\title{
Digestive physiology of pigs 2018
}

\author{
E. Roura', M. D. Lindemann ${ }^{2}$ and F. R. Dunshea ${ }^{3 \dagger} \oplus$ \\ ${ }^{1}$ Centre for Nutrition and Food Sciences, The University of Queensland, Saint Lucia, QLD 4069, Australia; ${ }^{2}$ Animal and Food Sciences, University of Kentucky, Lexington, \\ KY 40546, USA; ${ }^{3}$ Faculty of Veterinary and Agricultural Sciences, The University of Melbourne, Parkville, VIC 3010, Australia
}

(Received 7 July 2019; Accepted 8 July 2019)

On behalf of the scientific and organising committees of the 14th International Symposium of the Digestive Physiology of Pigs, we are delighted to introduce this special topic of Animal. This symposium has been the main forum where the scientific and technical developments in digestive physiology of pigs have been discussed on a triennial basis since 1979. Australia was proud to be the host country for the symposium which was held in Brisbane in August 2018, the first to be ever been held in Asia-Pacific. The symposium sessions focused not just on issues of importance for pig production but also on human health as the pig is increasingly being used as a biomedical model, in particular when studying diseases of the gastrointestinal tract (GIT).

As expected, the themes of the symposium were centred on the digestive system, focusing on specific aspects such as the emerging areas of the microbiota and the nutrient chemosensory mechanisms in the pig GIT. The role of the microbiota in gut and other health is topical and anyone with a passing interest in human or animal health has heard of the microbiota. Indeed, many of the submitted papers presented at the symposium and published in Advances in Animal Biosciences (2018) were devoted to characterising the microbiome in pigs but with very few insights into the regulation of the microbiota. However, understanding the nutritional and other factors that can impact on the microbiota has proven to be elusive; and in an elegant paper, Lyte and Lyte (2019) talk about the role the neuroendocrine system and neurotransmitters may play in the regulation of the microbiome. A theme throughout their paper is the interaction between stress, stress hormones and the microbiota and the complex feedback mechanisms that are operating.

Val-Laillet (2019) continued the theme of the complex interactions between the gut and brain; and over a decade, in vivo brain imaging studies have collated to present some fascinating insights about the gut-brain axis. They explain how the brain responds to food signals including preferred and aversive flavours or basic tastants such as sweet and bitter. Another fascinating area of their research is the impact

\footnotetext{
${ }^{\dagger}$ E-mail: fdunshea@unimelb.edu.au
}

of weight gain and obesity on the brain activity and metabolism and they draw parallels with obese human patients.

Roura et al. (2019) have summarised our current understanding on the sensing of nutrients in the GIT, describing the network of receptors and transceptors associated with the entero-endocrine system and gut peptide secretion (i.e. cholecystokinin, peptide tyrosine, glucagon-like peptide 1 or ghrelin). In addition, the mammalian chemosensory system has also been identified as a target for bacterial metabolites that stimulate nutrient receptors and trigger host cellular functions. Finally, a prolific area in chemosensing research refers to the studies of genetic polymorphisms associated with food choices and appetite. While most of the current knowledge has been obtained in laboratory rodents and humans, the review highlights the progress in pigs.

Piglet intestinal health is a major health concern in swine production and plays a critical role in the whole growth and development and in particular around weaning. Numerous potential stress factors around weaning can induce intestinal dysfunction in piglets, and Ji et al. (2019) review some of the nutritional interventions such as functional amino acids and, in particular, glutamine that may assist during the weaning transition. Humphrey et al. (2019) also focused their review on the impact of weaning on intestinal function and in particular intestinal barrier function and immunity. They hone in on practical and scalable nutritional approaches to influence intestinal barrier function and immunity through dietary lipid, protein and antioxidant manipulations.

The small intestine is the primary site for enzyme digestion of macronutrients in pigs; and for each macronutrient, digestion can start in the mouth (starch) or stomach (protein, triglyceride) but mostly occurs in the small intestine with catalysis by pancreatic enzyme secretions. Ratanpaul et al. (2019) focused on the likely rate-limiting steps in converting macronutrients into absorbable small molecules which are the access of enzymes for macronutrient substrates, enzyme activity levels available for digestion and uptake of products across the mucus layer that covers the epithelial cells. In particular, they focus on the roles of both soluble and insoluble dietary fibre components as modulators of each potential rate-limiting step. Ratanpaul et al. (2019) discuss the implications of these rate-limiting processes for both pig production and human health. 
We are sure that the reader will find that this series of reviews will provide many insights into the latest developments in digestive physiology of pigs and will appreciate the implications of this work for pig production specifically, livestock production in general and human health.

\section{Acknowledgements}

The authors wish to acknowledge the Scientific and Organising committees of Digestive Physiology of Pigs 2018 for their tireless efforts in organising the conference and program. The assistance of all who reviewed, submitted and invited papers is also appreciated. Finally, the authors would like to express their sincere gratitude to the symposium sponsors.

D F. R. Dunshea 0000-0003-3998-1240

\section{Declaration of interest}

None.

\section{Ethics statement}

None.

\section{Software and data repository resources}

None.

\section{References}

Advances in Animal Biosciences 2018. Proceedings of the 14th international symposium on digestive physiology of pigs (DPP2018), August 21-24 2018, Brisbane, Australia. Advances in Animal Biosciences 9, S61-S207. doi:10.1017/S2040470018000225

Humphrey B, Zhao J and Faris R 2019. Review: Link between intestinal immunity and practical approaches to swine nutrition. Animal 13, 2736-2744.

Ji FJ, Wang LX, Yang HS, Hu A and Yin Y 2019. Review: The roles and functions of glutamine on intestinal health and performance of weaning pigs. Animal 13 2727-2735.

Lyte JM and Lyte M 2019. Review: Microbial endocrinology: intersection of microbiology and neurobiology matters to swine health from infection to behaviour. Animal 13, 2689-2698.

Ratanpaul V, Williams BA, Blac, JL and Gidley MJ 2019. Review: Effects of fibre, grain starch digestion rate and the ileal brake on voluntary feed intake in pigs. Animal 13, 2745-2754.

Roura E, Depoortere I and Navarro M 2019. Review: Chemosensing of nutrients and non-nutrients in the human and porcine guts. Animal 13, 2714-2726.

Val-Laillet D 2019. Review: Impact of food, gut-brain signals, and metabolic status on brain activity in the pig model -10 years of nutrition research using in vivo brain imaging. Animal 13, 2699-2713. 